\title{
INFLAMMATORY BIOMARKERS AND BRAIN HEALTH INDICATORS IN CHILDREN WITH OVERWEIGHT AND OBESITY: THE ACTIVEBRAINS PROJECT
}

Mireia Adelantado-Renau, ${ }^{1}$, Irene Esteban-Cornejo ${ }^{2,3}$, María Rodriguez-Ayllon ${ }^{2}$, Cristina Cadenas-Sanchez ${ }^{2}$, Jose Juan Gil-Cosano², Jose Mora-Gonzalez ${ }^{2}$, Patricio Solis-Urra ${ }^{2,4}$, Juan Verdejo-Román ${ }^{5,6}$, Concepción M. Aguilera ${ }^{7,8}$, María Victoria Escolano-Margarit ${ }^{9}$, Antonio Verdejo-Garcia ${ }^{10}$, Andrés Catena ${ }^{11}$, Diego MolinerUrdiales $^{1}$ and Francisco B. Ortega ${ }^{2,12}$

${ }^{1}$ LIFE Research Group, University Jaume I, Castellon, Spain

${ }^{2}$ PROFITH "PROmoting FITness and Health through physical activity" research group, Sport and Health University Research Institute (iMUDS), Department of Physical and Sports Education, Faculty of Sport Sciences, University of Granada, Granada, Spain

${ }^{3}$ Center for Cognitive and Brain Health, Department of Psychology, Northeastern University, Boston, MA, USA

${ }^{4}$ IRyS Research Group. School of Physical Education. Pontificia Universidad Católica de Valparaíso, Valparaiso, Chile

${ }^{5}$ Laboratory of Cognitive and Computational Neuroscience (UCM-UPM), Centre for Biomedical Technology (CTB), Madrid, Spain

${ }^{6}$ Mind, Brain, and Behavior Research Center-CIMCYC, University of Granada, Granada, Spain 
${ }^{7}$ Department of Biochemistry and Molecular Biology II, Institute of Nutrition and Food Technology, Center for Biomedical Research, University of Granada, Granada. Spain

${ }^{8}$ CIBER Fisiopatología de la Obesidad y la Nutrición (CIBEROBN), Madrid, Spain; Instituto de Investigación Biosanitaria ibs. Granada, Spain

${ }^{9}$ Hospital Universitario San Cecilio. Granada. Spain

${ }^{10}$ Monash Institute of Cognitive and Clinical Neurosciences, Monash University, Melbourne, Australia

${ }^{11}$ Department of Experimental Psychology, Mind, Brain and Behavior Research Center (CIMCYC), University of Granada, Granada, Spain

${ }^{12}$ Department of Biosciences and Nutrition, Karolinska Institutet, Huddinge, Sweden.

Corresponding author: Mireia Adelantado-Renau, email address: adelantm@uji.es; postal address: Human Sciences Faculty, Department of Education, University Jaume I, Av. Vicent Sos Baynat, PC 12071, Castellon, Spain. Telephone number: 0034964 729840; Fax: 0034964 729264. ORCID ID: 0000-0001-6124-8876

\section{FUNDING}

This study was supported by the Spanish Ministry of Economy and Competitiveness (DEP2013-47540, DEP2016-79512-R and DEP2017-91544-EXP), the European Regional Development Fund (ERDF), the European Commission (No 667302) and the Alicia Koplowitz Foundation. This study was partially funded by the University of Granada, Research and Knowledge Transfer Fund (PPIT) 2016, the Excellence Actions Programme: Units of Excellence; Scientific Unit of Excellence on Exercise and Health 
(UCEES), the Andalusian Regional Government, the Regional Ministry of Economy, Knowledge, Entreprises and University, and the European Regional Development Fund (ERDF); ref. SOMM17/6107/UGR. In addition, this study was further supported by the SAMID III network, RETICS, funded by the PN I+D+I 2017-2021 (Spain). M.A.R is supported by a Predoctoral Research Grant from the University Jaume I (PREDOC/2015/13 and E-2017-13). I.E.C is supported by the Spanish Ministry of Economy and Competitiveness (IJCI-2017-33642). C.C.S is supported by the Government of Andalusian, Integrated Territorial Initiative 2014-2020 for the province of Cádiz (PI-0002-2017). J.M.G, is supported by the Spanish Ministry of Education, Culture and Sport (FPU14/06837). J.V.R is supported by a grant from the Spanish Ministry of Science, Innovation and Universities (FJCI-2017-33396). A.V.G is funded by a CDF fellowship of the Australian Medical Research Future Fund (MRF1141214) and his obesity research funded by NHMRC Project Grant APP1140197.

The authors declare that they have no conflict of interest. 
1 INFLAMMATORY BIOMARKERS AND BRAIN HEALTH INDICATORS IN

2 CHILDREN WITH OVERWEIGHT AND OBESITY: THE ACTIVEBRAINS

3 PROJECT

\section{ABSTRACT}

5 Introduction. Chronic inflammation plays an important role on the pathogenesis of

6 several cardiovascular and metabolic diseases, as well as on brain function and

7 behaviour. The aim of the present study was to examine the associations between

8 inflammatory biomarkers and a wide range of brain health indicators (i.e., academic

9 performance, executive function, behavioural and emotional functioning, and brain volume) in children with overweight/obesity.

Methods. A total of 107 children $(10.0 \pm 1.1$ years, $41 \%$ girls $)$ from the ActiveBrains project were included in the analysis. Five inflammatory biomarkers were analysed in plasma: white blood cell (WBC) count, interleukin-6 (IL-6), interleukin-1 $\beta$, tumor necrosis factor- $\alpha$ (TNF- $\alpha$ ), and C-reactive protein (CRP). Academic performance was assessed by Woodcock-Muñoz Tests of Achievement. Executive function was assessed through the Design Fluency Test for cognitive flexibility, the Stroop test for cognitive inhibition, and the Delayed Non-Match-to-Sample task for working memory. Behavioural and emotional functioning was evaluated through the Behavior Assessment System for Children (BASC) questionnaire. Total and regional brain volume was assessed by magnetic resonance imaging.

Results. IL-6 was inversely associated with adaptive skills $(\beta=-0.228 ; p=0.030)$, while TNF- $\alpha$ was related to mathematics $(\beta=-0.198 ; \mathrm{p}=0.034)$. In addition, CRP was positively associated with externalizing $(\beta=0.246 ; \mathrm{p}=0.046)$ and internalizing problems $(\beta=0.234 ; p=0.039)$, as well as the behavioural symptoms index $(\beta=0.236 ; p=0.047)$. 
However, these significant associations disappeared after multiple comparisons correction. Inflammatory biomarkers were not associated with executive function and total brain volumes. Regarding regional brain analyses, WBC was positively associated with gray matter volume in the left middle temporal gyrus $(\beta=0.387 ; p<0.001, k=44)$, and CRP was positively associated with gray matter volume in the right superior temporal gyrus $(\beta=0.439 ; \mathrm{p}<0.001, \mathrm{k}=29)$. Additionally, when adjusting by total brain volume, CRP was positively associated with gray matter volume in the right supplementary motor cortex $(\beta=0.453 ; \mathrm{p}<0.001, \mathrm{k}=51)$. Moreover, both, IL-6 $(\beta=0.366$; $\mathrm{p}<0.001, \mathrm{k}=81)$ and TNF- $\alpha(\beta=0.368 ; \mathrm{p}<0.001, \mathrm{k}=62)$ were positively associated with white matter volume around the right inferior frontal gyrus pars opercularis, while CRP was inversely associated with white matter volume around the left superior frontal gyrus $(\beta=-0.482 ; \mathrm{p}<0.001, \mathrm{k}=82)$. After adjusting by total brain volume, CRP was also inversely associated with white matter volume in 3 additional clusters ( $\beta$ ranging from -0.473 to $-0.404 ; \mathrm{p}<0.001, \mathrm{k}=87)$.

Conclusions. Inflammation was slightly associated with brain health (i.e., academic performance, behavioural and emotional functioning and regional brain volume) in children with overweight or obesity. Further larger longitudinal and interventional studies are warranted to elucidate the short-term and long-term effect of systemic lowgrade inflammation on children's brain health.

44 Keywords: inflammation, school performance, cognition, adaptive functioning, mental health, brain structure. 


\section{INTRODUCTION}

Childhood obesity has increased steadily in the past three decades becoming a serious worldwide health issue, with a prevalence rate of $23.8 \%$ of boys and $22.6 \%$ of girls in developed countries, and $12.9 \%$ of boys and $13.4 \%$ of girls in developing countries (Ng et al., 2014). Apart from weight gain, obesity has been closely linked to a cluster of disorders known as metabolic syndrome, resulting in subsequent systemic low-grade inflammation (Lumeng \& Saltiel, 2011). Besides chronic low-grade inflammation plays an important role on the pathogenesis of several cardiovascular and metabolic diseases such as atherosclerosis, diabetes, autoimmune diseases, and cancer (Hotamisligil, 2006; Libby, 2006), there is emerging evidence suggesting an association between inflammation and brain health, including cognitive, behavioural and emotional functioning (Slopen, Kubzansky, \& Koenen, 2013), as well as brain structure and function (Borsini, Zunszain, Thuret, \& Pariante, 2015).

Inflammatory biomarkers circulating in blood could access the central nervous system through different pathways, which might affect brain health. Particularly, cytokines may cross the blood-brain barrier (BBB) via active transport mechanisms or via vagal nerve stimulation (Banks, Lynch, \& Price, 2009). In addition, within the brain, inflammatory biomarkers could be expressed by astroglia, microglia, neurons and endothelial cells (Dantzer, O’Connor, Freund, Johnson, \& Kelley, 2008). Indeed, in both, humans and animal models, overexpression of peripheral pro-inflammatory biomarkers has been associated with impaired synaptic plasticity, neurogenesis and neuromodulation (McAfoose \& Baune, 2009), which in turn, may affect cognitive (e.g., reduction of spatial learning and memory skills), behavioural and emotional functioning, as well as brain structure (e.g., gray matter atrophy and lower tissue microstructure) (Borsini et al., 2015; Yirmiya \& Goshen, 2011). 
Prior research examining the relationship between peripheral inflammatory

biomarkers and brain health indicators in humans have mainly focused on the two endpoints of the lifespan (i.e., preterm and elderly populations). For instance, in preterm infants, perinatal inflammation has been associated with both, structural (e.g. risk of white matter damage) and functional brain alterations (e.g. diparesis, and impaired cognitive functioning, mental and motor development) (Kuban et al., 2017, 2015; O'Shea et al., 2013; Rose, Vassar, Cahill-Rowley, Hintz, \& Stevenson, 2015; Voltas et al., 2017). In elderly populations, inflammation has been closely related to neurodegenerative disorders such as Alzheimer's disease, dementia, and cognitive decline (Sartori, Vance, Slater, \& Crowe, 2012), behavioural disorders (Rosenblat, Cha, Mansur, \& McIntyre, 2014), and even with brain damage (Frodl \& Amico, 2014).

Evidence in healthy midlife adults has shown an inverse association of inflammatory biomarkers with executive function (e.g., cognitive inhibition, working memory and attention) (Windham et al., 2014), behavioural and emotional functioning (Marteinsdottir, Ernerudh, Jonasson, Kristenson, \& Garvin, 2016), and brain volume (Marsland, Gianaros, Abramowitch, Manuck, \& Hariri, 2008; Marsland et al., 2015). However, in normal developing children and adolescents, the limited research available has only indicated that inflammatory biomarkers may negatively influence academic performance (Esteban-Cornejo et al., 2016), cognitive function (Cullen et al., 2017) and intelligence (Lee et al., 2016). Thus, further studies investigating the association of inflammation and brain health, including academic performance, cognition, behavioural and emotional factors, and brain measurements in children are needed.

Given that inflammation is one of the earliest consequences of obesity, which in turn, has also shown to alter brain health in children (AL Miller, Jong, \& Lumeng, 2015; Sanders, Han, Baker, \& Cobley, 2015), examining the influence of inflammation 
96 on brain health indicators in the context of childhood obesity is of paramount

97 importance. To the best of our knowledge, this is the first study examining the influence

98 of inflammation on a wide range of brain health indicators, including gray and white

99 matter volumes in children. Thus, the aim of the present study was to examine the

100 associations of inflammatory biomarkers with brain health indicators (i.e., academic

101 performance, executive function, behavioural and emotional functioning, and total and

102 regional brain volume) in children with overweight/obesity. 


\section{METHODS}

\section{Participants}

The present cross-sectional study is part of the ActiveBrains project (http://profith.ugr.es/activebrains), a randomized controlled trial aimed to analyse the effects of an exercise program on brain, cognitive and academic performance, as well as on selected physical and mental health outcomes in children with overweight/obesity. Additional information about the methodology of the project can be found elsewhere (Cadenas-Sánchez et al., 2016). The results presented in this cross-sectional analysis belong to the baseline data obtained between November 2014 and February 2016. We estimated that a sample of 100 participants would be required to provide statistical power of $80 \%$ with a level of significance of 0.05 , assuming a dropout rate of $10 \%$. All participants were recruited from schools and university hospitals of Granada (southern Spain). The final sample included 107 children with overweight or obesity $(10.0 \pm 1.1$ years old; $41 \%$ girls) who had at least valid data for one inflammatory biomarker and one brain health indicator.

Parents or guardians were informed of the nature and characteristics of the study, and all signed an informed written consent. The ActiveBrains project was approved by the Human Research Ethics Committee of the University of Granada and was registered in ClinicalTrials.gov (identifier: NCT02295072).

\section{Inflammatory biomarkers}

After an overnight fast (at least $12 \mathrm{~h}$ ), blood samples were drawn from the antecubital vein. Blood samples in tubes containing EDTA were spun immediately at $1000 \mathrm{~g}$ for $10 \mathrm{~min}$. Plasma was isolated and stored at $-80^{\circ} \mathrm{C}$ until analysis in the Center of Biomedical Research (Granada, Spain). Five key inflammatory biomarkers analysed 
127 in plasma were included in this study: white blood cell (WBC, $10^{3} / \mu \mathrm{L}$ ) count, interleukin-6 (IL-6, pg/mL), interleukin-1 $(\mathrm{IL}-1 \beta, \mathrm{pg} / \mathrm{mL})$, tumor necrosis factor- $\alpha$ (TNF- $\alpha, p g / m L)$, and C-reactive protein (CRP, mg/L). WBC count was analysed with automated blood cell counters. IL-6, IL-1 $\beta$ and TNF- $\alpha$ were quantified by multiple analyte profiling technology (MILLIPLEX ${ }^{\circledR}$ MAP Human High Sensitivity T Cell Magnetic Bead Panel, EMD Millipore Corporation, Missouri, U.S.A.) using a kit plex (HCYIL6-MAG Anti-Human IL-6 Beads set, HCYIL1B-MAG Anti-Human IL-1 $\beta$ Bead, and HCYTNFA-MAG Anti-Human TNF $\alpha$ Beads set). The intra- and inter-assay precision coefficients of variation (CVs) for IL-6 were 5\% and 20\%, respectively, and sensitivity was $0.11 \mathrm{pg} / \mathrm{mL}$. For both, IL- $1 \beta$ and TNF- $\alpha$ the intra- and inter-assay precision CVs were 5\% and $15 \%$, respectively, with a sensitivity of $0.14 \mathrm{pg} / \mathrm{mL}$ for IL-

\section{Academic performance}

Academic performance was assessed by the Spanish version of the Batería III Woodcock-Muñoz Tests of Achievement, which has shown a high reliability and validity (McGrew \& Woodcock, 2001). Thirteen tests were individually administered in one session of 100-120 $\mathrm{min}$, and the obtained data were processed using the Compuscore and profile software version 3.1 (Riverside Publishing Company, Itasca, IL, USA). For the current study, a standard T-score based on an average of 100 and standard deviations of 15 points was obtained for the following broad academic performance indicators: mathematics (including calculation skills, problem solving and the ability to subtract, sum, multiply or divide quickly), reading (including word identification, reading speed and comprehension), writing (including spelling, quality of written sentences and speed of writing), and total achievement (including mathematics, reading and writing). 
Executive function

The assessment of executive function was conducted individually for each child, and lasted approximately 45-60 min. Three main indicators were assessed: cognitive flexibility, cognitive inhibition and working memory.

Cognitive flexibility and cognitive inhibition were assessed using two paperpencil based sub-tests of the Delis-Kaplan Executive Function System (D-KEFS) (Delis, Kaplan, \& Kramer, 2001). For cognitive flexibility, we used the Design Fluency Test (DFT) (Delis et al., 2001), in which participants should connect dots using only four straight lines to design as many novel shapes as possible in periods of 60 seconds. The total number of correct drawn designs was registered and used in the analysis. For cognitive inhibition, we used a modified version of the Stroop test (Stroop, 1935) called the Stroop Colour Word Test, which includes measurements of 1) fundamental linguistic skills (i.e., namely speed of naming), and 2) inhibition, where colour-words are printed in a colour that differs from their meaning, and the task consists of naming the colour of the word and avoiding its reading. An interference score was obtained by subtracting completion times (2-1) (Moreno-López, Soriano-Mas, Delgado-Rico, RioValle, \& Verdejo-García, 2012). Because the Stroop interference scores are inversely related to cognitive inhibition, it was multiplied by -1 .

Working memory was measured using a modified version of the Delayed NonMatch-to-Sample (DNMS) computerized task, that was previously developed to differentiate between manipulation (high memory load) and maintenance (low memory load) cognitive processes (Robinson et al., 2009). Each trial was presented on a computer screen using E-Prime and consisted of two phases: sample and choice. Sixteen practice trials plus 140 experimental trials were randomly presented. Participants had to remember 4 Pokémon cartoons (i.e., sample phase), and subsequently, to select the 
177 cartoon that had not previously appeared (i.e., choice phase) between two different

targets. In the high memory load condition, in which 4 different stimuli were presented before the choice phase, reaction time and response accuracy were registered. A ratio of working memory was calculated as the quotient between reaction time and response accuracy, and for analytic purposes, this ratio was multiplied by -1 , so higher ratio indicates better working memory.

\section{Behavioural and emotional functioning}

The Behavior Assessment System for Children (BASC), level-2 for children aged 6-12 years old, which has shown extensive psychometric properties in both nonreferred and clinical populations with reliabilities for the subscales ranging from 0.80 to 0.87, was completed by parents to assess negative behaviours and positive attributes (Reynolds \& Kamphaus, 2004). BASC responses cluster into 4 global dimensions of behavioural and emotional functioning: externalizing problems (including aggressivity, hyperactivity and behavioural problems), internalizing problems (including anxiety, depression and somatization), adaptive skills (including adaptability, social skills and leadership) and a behavioural symptoms index (including aggressivity, hyperactivity, attention problems, atypical behaviours, anxiety and depression). For each indicator, standard T-scores with an average of 50 and standard deviations of 10 points were used in the analyses.

Magnetic Resonance Imaging (MRI) procedure

All images were collected on a 3.0 Tesla Siemens Magnetom Tim Trio scanner (Siemens Medical Solutions, Erlangen, Germany) equipped with a 32-channel head coil. High-resolution, T1-weighted images were acquired using a 3D MPRAGE (Magnetization-Prepared Rapid Gradient-Echo) sequence. Acquisition parameters were: 
repetition time $(\mathrm{TR})=2300 \mathrm{~ms}$, echo time $(\mathrm{TE})=3.1 \mathrm{~ms}$, inversion time $(\mathrm{TI})=900 \mathrm{~ms}$, flip angle $=9^{\circ}$, field of view $($ FOV $)=256 \times 256$, acquisition matrix $=320 \times 320,208$ slices, resolution $=0.8 \times 0.8 \times 0.8 \mathrm{~mm}$, and scan duration of 6 min and $34 \mathrm{~s}$.

Imaging data were pre-processed using the Statistical Parametric Mapping

software (SPM 12; Wellcome Department of Cognitive Neurology, London, UK) implemented in Matlab (The MathWorks, Inc, Natick, MA). Information about preprocessing steps has been previously detailed (Esteban-Cornejo et al., 2017). First, using the latest segmentation algorithm implemented in SPM12, we segmented T1weighted structural images of each participant into gray matter tissue, white matter tissue, and cerebrospinal fluid (Ashburner \& Friston, 2005). Second, segmented gray matter/white matter tissues for all participants were used to generate a customized template using Diffeomorphic Anatomical Registration Through Exponentiated Lie algebra (DARTEL) (Ashburner, 2007). DARTEL estimates a best set of smooth deformations from each participant's tissue to their common average and reiterates the process until convergence. The resulting images were spatially normalized to Montreal Neurological Institute (MNI) space with affine transformation to create the DARTEL template. Subsequently, each participant's segmented images were normalized to the DARTEL template via nonlinear transformation. In order to perform a volume change correction, the normalized gray matter images were modulated with Jacobian determinants derived from the spatial normalization (Ashburner \& Friston, 2000). Finally, the volumetric images were smoothed by convolving them with an isotropic Gaussian kernel of $8 \mathrm{~mm}$ full-width at half-maximum (FWHM). Total gray and white matter volumes were calculated from the non-normalized segmented images. Total brain volume (TBV) was calculated by adding the volumes of gray and white matter. 
Sex, peak height velocity (PHV), parental education level, body mass index

227 (BMI) and TBV were included as covariates.

PHV was obtained from weight, height and seated height using Moore's equations (Moore et al., 2015). Years from PHV were calculated from the chronological age, and the difference in years was used in the analysis as a value of maturation.

Parental education level was used as a proxy of socioeconomic status. Parent responses were combined as: neither of the parents had a university degree, one of the parents had a university degree and both parents had a university degree.

Body weight was measured to the nearest $0.1 \mathrm{~kg}$ using an electronic scale (SECA 861, Hamburg, Germany) lightly dressed and without shoes. Height was measured to the nearest $0.1 \mathrm{~cm}$ using a wall-mounted stadiometer (SECA 225, Hamburg, Germany). Measures were assessed in duplicate and average measures were used for data analysis. BMI was calculated as weight/height square $\left(\mathrm{kg} / \mathrm{m}^{2}\right)$ and BMI categories were defined (i.e. overweight, obesity grade I, II, III) according to age- and sex-specific BMI cut-off points (Cole \& Lobstein, 2012).

\section{Statistical analysis}

Descriptive characteristics are presented as mean and standard deviations or percentages. All variables were checked for normality using both graphical (normal probability plots) and statistical (Kolmogorov-Smirnov test) procedures. Due to its skewed distribution, CRP, IL-6, TNF- $\alpha$, IL-1 $\beta$, cognitive inhibition and working memory ratio were normalized using Blom's formula before analysis (Blom, 1958). As preliminary analyses showed no significant interactions of sex with inflammatory biomarkers in relation to brain health indicators (all $\mathrm{p}>0.10$ ), all analyses were performed for the whole sample. 

biomarkers with academic performance, executive function, behavioural and emotional functioning and total brain volumes adjusting for sex, PHV, parental education level, and BMI. We conducted the Benjamini-Hochberg correction for assessing multiple comparisons between inflammatory biomarkers and brain health indicators. Briefly, this method uses ranked p-values to determine the cut-off, at which point the Type-I error rate is below 0.05 (Benjamini \& Hochberg, 1995). All the analyses were performed using the IBM SPSS Statistics for Windows version 22.0 (Armonk, NY: IBM Corp), and the level of significance was set at $\mathrm{P}<0.05$.

Statistical analyses of imaging data were performed using the General Lineal Model approach implemented in SPM12. The association of each inflammatory biomarker with gray matter volume was examined using five whole-brain voxel-wise multiple regression models (one for each inflammatory biomarker), adjusted for sex, PHV, parental education level and BMI. Additional analyses were conducted including coordinates of each significant cluster.

The statistical threshold in the imaging analyses was calculated with AlphaSim, as implemented in Resting-State fMRI Data Analysis Toolkit toolbox (RESTplus) (Song et al., 2011). Parameters were defined as follows: cluster connection radius $(\mathrm{rmm})=5 \mathrm{~mm}$ and the actual smoothness of the data after model estimation, incorporating a gray mask volume of 128190 voxels or a white matter mask volume of 302567 voxels, respectively. The voxel-level alpha significance (threshold, $\mathrm{p}<0.001$ uncorrected) along with the appropriate cluster size for controlling for multiple comparisons in each analysis were indicated in the results. The resulting cluster extents 
275 were further adjusted to account for the non-isotropic smoothness of structural images,

276 in accordance with Hayasaka et al. (2004). 


\section{RESULTS}

Descriptive characteristics of the study population, including inflammatory

279 biomarkers and brain health indicators, are presented in Table 1. Participants showed a BMI of $26.7 \mathrm{~kg} / \mathrm{m}^{2}$, being $26 \%$ overweight and $74 \%$ obese. Overall, boys and girls showed similar values of inflammatory biomarkers. Academic performance ranged from 102.1 to $114.2( \pm \sim 12)$, cognitive flexibility scored 20.0, cognitive inhibition scored 40.6, and working memory ratio was 15.2. In addition, participants presented behavioural and emotional functioning indicators ranging from 49.0 to 54.4 , and a total brain volume of $1199 \mathrm{~cm}^{3}$ (793 $\mathrm{cm}^{3}$ of gray matter).

The results of the multiple linear regression models showing the associations of inflammatory biomarkers with brain health indicators after adjustment for sex, PHV, parental education level, and BMI are shown in Table 2. IL-6 was inversely associated with adaptive skills $(\beta=-0.228 ; \mathrm{p}=0.030)$, and TNF- $\alpha$ was inversely related to mathematics $(\beta=-0.198 ; p=0.034)$. In addition, CRP was positively associated with externalizing $(\beta=0.246 ; p=0.046)$ and internalizing problems $(\beta=0.234 ; p=0.039)$, as well as, with the behavioural symptoms index $(\beta=0.236 ; p=0.047)$. However, these significant associations disappeared after multiple comparisons correction. Inflammatory biomarkers were not associated with executive function and total brain volumes.

Figure 1 displays the brain regions showing positive associations between inflammatory biomarkers and gray matter volume after adjustment for sex, PHV, parental education level and BMI. WBC was positively associated with gray matter volume in the left middle temporal gyrus $(\beta=0.387, \mathrm{t}=4.20 ; \mathrm{p}<0.001, \mathrm{k}=44$; Figure $1 \mathrm{~A})$. In addition, CRP was positively associated with gray matter volume in the right superior temporal gyrus $(\beta=0.439, \mathrm{t}=4.37 ; \mathrm{p}<0.001, \mathrm{k}=29$; Figure 1B). IL-6, IL-1 $\beta$ and TNF- $\alpha$ 
were not positively associated with regional gray matter volume. Furthermore, there were no statistically significant inverse associations between any inflammatory biomarker and gray matter volume in any region of the brain. Similar results were found after adjusting by TBV (Table S1). Additionally, CRP was positively associated with gray matter volume in the right supplementary motor cortex $(\beta=0.453, \mathrm{t}=3.88 ; \mathrm{p}<0.001$, $\mathrm{k}=51)$.

Figure 2 presents the brain regions showing positive and inverse associations between inflammatory biomarkers and white matter volume after adjustment for potential confounders. IL-6 $(\beta=0.366, \mathrm{t}=4.00 ; \mathrm{p}<0.001, \mathrm{k}=81)$ and TNF- $\alpha(\beta=0.368$, $\mathrm{t}=3.98 ; \mathrm{p}<0.001, \mathrm{k}=62$ ) were positively associated with white matter volume around the right inferior frontal gyrus pars opercularis (Figure 2A). CRP was inversely associated with white matter volume around the left superior frontal gyrus $(\beta=-0.482, \mathrm{t}=-5.11$; $\mathrm{p}<0.001, \mathrm{k}=82$; Figure $2 \mathrm{~B}$ ). Furthermore, there were no other statistically significant positive or inverse associations between any inflammatory biomarker and white matter volume in any region of the brain. Results persisted after including TBV as a covariate (Table S2). Furthermore, CRP was inversely associated with white matter volume bilaterally around the superior frontal gyrus pars orbital $(\beta=-0.404, t=-3.76$ and $\beta=-0.473, t=-4.17 ; p<0.001, k=87)$, and around the right middle cingulum $(\beta=-0.424$, $\mathrm{t}=-3.77 ; \mathrm{p}<0.001, \mathrm{k}=87)$. 
The main findings of the present study indicated that inflammatory biomarkers were slightly associated with brain health indicators in children with overweight/obesity. Higher levels of IL-6 and TNF- $\alpha$ were associated with lower adaptive skills and mathematics, respectively, while CRP was positively associated with externalizing and internalizing problems, as well as with the behavioural symptoms index. However, these significant associations disappeared after correcting for multiple comparisons. In addition, inflammation was not associated with executive function and total brain volumes. Unexpected, a whole-brain analytical approach revealed that higher levels of WBC and CRP were associated with greater regional gray matter volume in the left middle temporal gyrus, and in the right superior temporal gyrus, respectively. Moreover, higher levels of IL- 6 and TNF- $\alpha$ were associated with greater white matter volume around the right inferior frontal gyrus pars opercularis, while CRP was the only inflammatory biomarker showing an expected and inverse association with white matter volume around the left superior frontal gyrus. These results contribute to the current knowledge by suggesting that inflammation, one of the earliest consequences of obesity, is not only associated with behavioural and emotional functioning, but also with regional brain volumes in children with overweight/obesity.

This is the first study that examines the association between inflammatory biomarkers and a wide range of brain health indicators, including academic performance, executive function, behavioural and emotional functioning, as well as total and regional brain volumes. Regarding academic performance, to date only one study has examined the association between inflammatory biomarkers and academic achievement in a sample of 494 healthy children and adolescents showing that WBC, IL-6 and CRP (but not TNF- $\alpha$ ) were inversely associated with school grades (i.e., math, 
language, the mean of math and language, and grade point average), independently of adiposity (Esteban-Cornejo et al., 2016). In this sense, we found an isolated association between TNF- $\alpha$ and mathematics, which disappeared after correcting for multiple comparisons. Therefore, the fact that previous research did not use multiple comparisons correction, along with differences in methodological issues (e.g., school grades vs. academic performance standardized questionnaire), and the multifactorial nature of academic performance which involves both, cognitive and non-cognitive traits, could partially explain these inconsistent findings. not to affect proliferation and gliogenesis, with no effects on cognitive functioning, TNF- $\alpha$ plays a substantial role in the inhibition of neurogenesis, which may affect cognition (Borsini et al., 2015). However, our results indicate a lack of association between inflammatory biomarkers and executive function in children with overweight/obesity. These findings are in consonance with previous interventional (Grigoleit et al., 2010) and prospective (Jonker et al., 2014) studies conducted in youths. For instance, Grigoleit et al. (2010) conducted an interventional study in healthy young men showing that the administration of lipopolysaccharides increased circulating neutrophils and plasma cytokine levels, without affecting memory performance, selective attention or executive function. Likewise, Jonker et al. (2014) showed that high sensitive-CRP in adolescents was not associated with memory and executive functioning two years later.

In contrast, an emerging body of literature has suggested an inverse association between inflammation and executive function in preterm infants (Kuban et al., 2017; O’Shea et al., 2013; Rose et al., 2015), children (Huang et al., 2016; Lee et al., 2016), adolescents (Cullen et al., 2017), and adult populations (Marsland et al., 2015; Sartori et 
al., 2012; Windham et al., 2014). In youths, no previous studies have focused on

population with overweight/obesity, which hinders comparisons among studies. In a study conducted in normal-weight children from impoverished countries, higher inflammation was closely linked to lower general intelligence, independently of nutritional and socioeconomic status (Lee et al., 2016). Another study conducted in normal-weight children, of which $60 \%$ presented obstructive sleep apnea, revealed that high levels of inflammatory biomarkers were related with decreased executive functions (Huang et al., 2016). In addition, a more recent research suggested that TNF- $\alpha$ and interferon- $\gamma$ (but not several interleukins) were inversely associated with memory in a general population of children (Kyriklaki et al., 2019). In adolescents, Cullen et al. (2017) found that salivary CRP was inversely related to letter fluency and cognitive inhibition, but not to memory. Collectively, although inflammatory biomarkers may also play a key role on cognitive processes in youths, the negative influence of inflammation on cognitive functioning seems to become more evident during the early and late stages of the human lifespan. Thus, differences in age, ethnicity population, as well as in the study design and methodologic technics (e.g., blood vs. salivary analysis), together with the fact that it is likely that our study was underpower to determinate statistically significant results, may be the responsible for the divergent results. Therefore, further studies in young populations are warranted to clarify the role of inflammation on cognition, and specifically in populations with overweight/obesity, since adiposity is a key factor on this relationship (AL Miller et al., 2015).

According to behavioural and emotional functioning, IL-6 was inversely associated with adaptive skills, while CRP was directly associated with externalizing and internalizing problems, as well as with the behavioural symptoms index in children with overweight/obesity. However, in our relatively small sample, these significant 
associations disappeared when correcting for multiple comparisons. These findings partially concur with prior research in infants (Voltas et al., 2017), children (Brambilla, Monteleone, \& Maj, 2004; Cicchetti, Handley, \& Rogosch, 2015; Slopen et al., 2013) and adolescents (Belem da Silva et al., 2017). For example, in children with major depressive disorders, although TNF- $\alpha$ was negatively correlated to depressive symptoms, IL-1 $\beta$ was positively correlated with both, depressive and anxiety symptoms (Brambilla et al., 2004). In addition, elevated levels of CRP were related to higher internalizing problems in recently maltreated children, but not in non-maltreated children (Cicchetti et al., 2015). However, the cross-sectional design of the abovementioned studies conducted in children and adolescents cannot determine causality, making reverse causation equally plausible; behavioural functioning could also influence inflammation. In fact, prior research showed that internalizing and externalizing problems at age 8 were associated with higher concentrations of IL-6 and CRP at age 10, respectively (Slopen et al., 2013). Interestingly, in another study, adolescents with internalizing behaviours presented higher levels of IL-6, when compared with their healthy peers (Belem da Silva et al., 2017). Thus, longitudinal and interventional studies examining the relationship between inflammation and behavioural functioning in young populations are needed to elucidate the direction of causality.

The reasons underlying why inflammatory biomarkers were associated with behavioural and emotional functioning cannot be elucidated in the present study. 416 Nevertheless, we suggested some mechanisms that could be implicated in this 417 association. First, several cytokines convert tryptophan to kynurenine, as well as amino compounds into acidic compounds, through different molecular processes reducing levels of serotonin (Rosenblat et al., 2014; Zhang, Terreni, De Simoni MG, \& Dunn, 2001), which may affect mood, and consequently, behavioural and emotional 
421 functioning. Second, cytokines can activate the hypothalamic-pituitary-adrenal axis, releasing specific hormones such as cortisol, an important component of the stress response (AH. Miller, Haroon, Raison, \& Felger, 2013), which might contribute to behavioural dysfunctions. Third, inflammatory biomarkers also activate microglia, which in turn, promotes apoptosis of functional neuronal pathways, leading to poor brain functioning, and behavioural and emotional problems (Ekdahl, 2012). Last, inflammation could affect brain structure and function through the impairment of neuroplasticity, which might also contribute to behavioural and emotional disorders (AH. Miller et al., 2013). Thus, we speculate that inflammation may influence specific molecular processes altering brain function, and possibly leading to behavioural and emotional dysfunctions. Importantly, to our knowledge this is the first neuroimaging research aimed to test the associations of inflammatory biomarkers with total brain volumes, and regional gray matter and white matter volumes using a whole-brain analytical approach, which hampers comparisons among studies. Our results revealed no association of inflammatory biomarkers with total gray matter, total white matter and total brain volume. Regarding the regional brain volumes findings, we found positive and negative isolated associations of inflammatory biomarkers with gray and white matter volumes in some small clusters. Surprisingly, higher concentrations of WBC and CRP were associated with greater gray matter volume in the left middle temporal gyrus, and in the right superior temporal gyrus, respectively. Additionally, when considering TBV, CRP was also positively associated with gray matter volume in the right supplementary motor cortex. Furthermore, higher concentrations of IL- 6 and TNF- $\alpha$ were associated with greater white matter volume around the right inferior frontal gyrus pars opercularis. Conversely, higher levels of CRP were associated with lower white matter 
volume in 1 cluster around the left superior frontal gyrus. After considering TBV, CRP was also inversely associated with white matter volume bilaterally around the superior frontal gyrus pars orbital and in the middle cingulum. Prior evidence from animal models has shown that brain morphology may be particularly vulnerable to inflammation-related processes (Yirmiya \& Goshen, 2011). In parallel to animal work, increased attention has been paid to the relationship between inflammation and brain structure in adult humans. Findings from a previous study in healthy midlife adults showed that higher levels of IL-6 were inversely associated with hippocampal gray matter volume, as well as, with gray matter volume in the medial prefrontal cortex and in the right cerebellum (Marsland et al., 2008). In a more recent study, both IL-6 and CRP showed inverse associations with cortical gray and white matter volumes, hippocampal volume, and cortical surface area (Marsland et al., 2015). In line with our findings, research in healthy elderly adults showed that higher levels of IL-6 and CRP were associated with greater white matter hyperintensities, and lower total gray matter and hippocampal volumes (Satizabal, Zhu, Mazoyer, Dufouil, \& Tzourio, 2012). Similarly, Taki et al. (2013) found an inverse association between high sensitivity-CRP and regional gray matter volume in the left temporal cortex, while Bettcher et al. (2012) showed an inverse association of CRP with left medial temporal lobe volumes. Thus, brain structure has shown to be particularly vulnerable to the effects of age.

We speculate that the few isolated and mixed associations between inflammation and regional brain volumes found in our study are probably due to the fact that in children brain is not as vulnerable as in preterm infants and older adults, and inflammation has not significant adverse effects on brain yet. In addition, the fact that inflammatory biomarkers at physiologically normal levels can act as both, anti- and proinflammatory substances, could explain the positive and inverse associations of 
471 inflammation with regional brain volume found in our study. Therefore, more studies

472 are needed to elucidate the developmental period at which inflammation produces 473 detrimental effects on brain structure, as well as, to assess the short-term and long-term 474 effect of systemic low-grade inflammation on brain.

\section{Limitations and strengths}

The current study has some limitations that must be mentioned. The crosssectional design of our analyses prevents us from inferring causal relationships. In addition, our analyses need replication in a larger sample size in order to elucidate the associations between inflammation and brain health in children with overweight/obesity. However, the strengths of the study comprise the inclusion of a wide range of brain health indicators assessed through validated tools, as well as the use of a whole-brain analytical approach.

\section{Conclusions}

Our findings reveal that inflammation is slightly associated with brain health, particularly with behavioural and emotional functioning, as well as with regional brain volumes, in children with overweight or obesity. Further larger longitudinal and

487 interventional studies in children with overweight/obesity are warranted to elucidate the pathways by which inflammation is linked to brain health, as well as the short-term and long-term effect of systemic low-grade inflammation and obesity on brain health.

Acknowledgements. The authors thank children and parents who participated in this 491 study.

492 Conflict of interest. The authors declare that they have no conflict of interest. 


\section{REFERENCES}

494

495

496

497

498

499

500

501

502

503

504

505

506

507

508

509

510

511

512

513

514

Ashburner, J. (2007). A fast diffeomorphic image registration algorithm. NeuroImage, 38(1), 95-113.

Ashburner, J., \& Friston, K. J. (2000). Voxel-based morphometry-the methods. NeuroImage, 11(6 Pt 1), 805-821.

Ashburner, J., \& Friston, K. J. (2005). Unified segmentation. NeuroImage, 26(3), 839851.

Banks, W. A., Lynch, J. L., \& Price, T. O. (2009). Cytokines and the Blood-Brain Barrier. In The Neuroimmunological Basis of Behavior and Mental Disorders (pp. 3-17). Boston, MA: Springer US. https://doi.org/10.1007/978-0-387-84851-8_1

Belem da Silva, C. T., de Abreu Costa, M., Kapczinski, F., de Aguiar, B. W., Salum, G. A., \& Manfro, G. G. (2017). Inflammation and internalizing disorders in adolescents. Progress in Neuro-Psychopharmacology and Biological Psychiatry, 77, 133-137. https://doi.org/10.1016/j.pnpbp.2017.03.023

Benjamini, Y., \& Hochberg, Y. (1995). Controlling the False Discovery Rate: A Practical and Powerful Approach to Multiple Testing. Journal of the Royal Statistical Society. Series B (Methodological), 57, 289-300. https://doi.org/10.2307/2346101

Bettcher, B. M., Wilheim, R., Rigby, T., Green, R., Miller, J. W., Racine, C. A., ... Kramer, J. H. (2012). C-reactive protein is related to memory and medial temporal brain volume in older adults. Brain, Behavior, and Immunity, 26(1), 103-108. https://doi.org/10.1016/j.bbi.2011.07.240 
Blom, G. (1958). Statistical estimates and transformed beta-variables (New York). John Wiley \& Sons.

Borsini, A., Zunszain, P. A., Thuret, S., \& Pariante, C. M. (2015). The role of inflammatory cytokines as key modulators of neurogenesis. Trends in Neurosciences, 38(3), 145-157. https://doi.org/10.1016/j.tins.2014.12.006

Brambilla, F., Monteleone, P., \& Maj, M. (2004). Interleukin-1 $\beta$ and tumor necrosis factor- $\alpha$ in children with major depressive disorder or dysthymia. Journal of Affective Disorders, 78(3), 273-277. https://doi.org/10.1016/S01650327(02)00315-4

Cadenas-Sánchez, C., Mora-González, J., Migueles, J. H., Martín-Matillas, M., GómezVida, J., Escolano-Margarit, M. V., ... Ortega, F. B. (2016). An exercise-based randomized controlled trial on brain, cognition, physical health and mental health in overweight/obese children (ActiveBrains project): Rationale, design and methods. Contemporary Clinical Trials, $\quad 47, \quad 315-324$. https://doi.org/10.1016/j.cct.2016.02.007

Cicchetti, D., Handley, E. D., \& Rogosch, F. A. (2015). Child maltreatment, inflammation, and internalizing symptoms: Investigating the roles of C-reactive protein, gene variation, and neuroendocrine regulation. Development and Psychopathology, 27(2), 553-566. https://doi.org/10.1017/S0954579415000152

Cole, T. J., \& Lobstein, T. (2012). Extended international (IOTF) body mass index cutoffs for thinness, overweight and obesity. Pediatric Obesity, 7(4), 284-294. https://doi.org/10.1111/j.2047-6310.2012.00064.x

Cullen, A. E., Tappin, B. M., Zunszain, P. A., Dickson, H., Roberts, R. E., Nikkheslat, N., ... Laurens, K. R. (2017). The relationship between salivary C-reactive protein 

and cognitive function in children aged 11-14 years: Does psychopathology have a moderating effect? Brain, Behavior, and Immunity, 66, 221-229. https://doi.org/10.1016/j.bbi.2017.07.002

Dantzer, R., O’Connor, J. C., Freund, G. G., Johnson, R. W., \& Kelley, K. W. (2008). From inflammation to sickness and depression: when the immune system subjugates the brain. Nature Reviews Neuroscience, 9(1), 46-56. https://doi.org/10.1038/nrn2297

Delis, D., Kaplan, E., \& Kramer, J. (2001). Delis-Kaplan Executive Function System (D-KEFS). San Antonio, TX: The Psychological Corporation.

Ekdahl, C. T. (2012). Microglial Activation - Tuning and Pruning Adult Neurogenesis. Frontiers in Pharmacology, 3, 41. https://doi.org/10.3389/fphar.2012.00041

Esteban-Cornejo, I., Cadenas-Sanchez, C., Contreras-Rodriguez, O., Verdejo-Roman, J., Mora-Gonzalez, J., Migueles, J. H., ... Verdejo-Garcia, A. (2017). A whole brain volumetric approach in overweight/obese children: Examining the association with different physical fitness components and academic performance. The ActiveBrains project. NeuroImage, 159 , $346-354$. https://doi.org/10.1016/j.neuroimage.2017.08.011

Esteban-Cornejo, I., Martinez-Gomez, D., Gómez-Martínez, S., del Campo-Vecino, J., Fernández-Santos, J., Castro-Piñero, J., ... Gómez-Gallego, F. (2016). Inflammatory biomarkers and academic performance in youth. The UP \& DOWN Study. Brain, Behavior, and Immunity, 54, 122-127. https://doi.org/10.1016/j.bbi.2016.01.010

Frodl, T., \& Amico, F. (2014). Is there an association between peripheral immune markers and structural/functional neuroimaging findings? Progress in Neuro- 
Psychopharmacology and Biological Psychiatry, 48, 295-303. https://doi.org/10.1016/j.pnpbp.2012.12.013

Grigoleit, J.-S., Oberbeck, J. R., Lichte, P., Kobbe, P., Wolf, O. T., Montag, T., ... Schedlowski, M. (2010). Lipopolysaccharide-induced experimental immune activation does not impair memory functions in humans. Neurobiology of Learning and Memory, 94(4), 561-567. https://doi.org/10.1016/j.nlm.2010.09.011

Hayasaka, S., Phan, K. L., Liberzon, I., Worsley, K. J., \& Nichols, T. E. (2004). Nonstationary cluster-size inference with random field and permutation methods. NeuroImage, 22(2), 676-687. https://doi.org/10.1016/j.neuroimage.2004.01.041

Hotamisligil, G. S. (2006). Inflammation and metabolic disorders. Nature, 444(7121), 860-867. https://doi.org/10.1038/nature05485

Huang, Y.-S., Guilleminault, C., Hwang, F.-M., Cheng, C., Lin, C.-H., Li, H.-Y., \& Lee, L.-A. (2016). Inflammatory cytokines in pediatric obstructive sleep apnea. Medicine, 95(41), e4944. https://doi.org/10.1097/MD.0000000000004944

Jonker, I., Klein, H. C., Duivis, H. E., Yolken, R. H., Rosmalen, J. G. M., \& Schoevers, R. A. (2014). Association between Exposure to HSV1 and Cognitive Functioning in a General Population of Adolescents. The TRAILS Study. PLoS ONE, 9(7), e101549. https://doi.org/10.1371/journal.pone.0101549

Kuban, K. C. K., Joseph, R. M., O’Shea, T. M., Heeren, T., Fichorova, R. N., Douglass, L., ... Extremely Low Gestational Age Newborn (ELGAN) Study Investigators. (2017). Circulating Inflammatory-Associated Proteins in the First Month of Life and Cognitive Impairment at Age 10 Years in Children Born Extremely Preterm. The Journal of Pediatrics, 180 116-123.e1. https://doi.org/10.1016/j.jpeds.2016.09.054 
Kuban, K. C. K., O’Shea, T. M., Allred, E. N., Fichorova, R. N., Heeren, T., Paneth, N., ... ELGAN Study Investigators. (2015). The Breadth and Type of Systemic Inflammation and the Risk of Adverse Neurological Outcomes in Extremely Low Gestation Newborns. Pediatric Neurology, 52(1), 42-48. https://doi.org/10.1016/j.pediatrneurol.2014.10.005

Kyriklaki, A., Margetaki, K., Kampouri, M., Koutra, K., Bitsios, P., Chalkiadaki, G., ... Chatzi, L. (2019). Association between high levels of inflammatory markers and cognitive outcomes at 4 years of age: The Rhea mother-child cohort study, Crete, Greece. Cytokine, 117, 1-7. https://doi.org/10.1016/j.cyto.2019.01.010

Lee, S. E., West, K. P., Cole, R. N., Schulze, K. J., Wu, L. S.-F., Yager, J. D., ... Christian, P. (2016). General intelligence is associated with subclinical inflammation in Nepalese children: A population-based plasma proteomics study. Brain, Behavior, and Immunity, 56, 253-263. https://doi.org/10.1016/j.bbi.2016.03.023

Libby, P. (2006). Inflammation and cardiovascular disease mechanisms. The American Journal of Clinical Nutrition, 83(2), 456S-460S. https://doi.org/10.1093/ajcn/83.2.456S

Lumeng, C. N., \& Saltiel, A. R. (2011). Inflammatory links between obesity and metabolic disease. Journal of Clinical Investigation, 121(6), 2111-2117. https://doi.org/10.1172/JCI57132

Marsland, A. L., Gianaros, P. J., Abramowitch, S. M., Manuck, S. B., \& Hariri, A. R. (2008). Interleukin-6 Covaries Inversely with Hippocampal Grey Matter Volume in Middle-Aged Adults. Biological Psychiatry, 64(6), 484-490. https://doi.org/10.1016/j.biopsych.2008.04.016 
Marsland, A. L., Gianaros, P. J., Kuan, D. C. H., Sheu, L. K., Krajina, K., \& Manuck, S. B. (2015). Brain morphology links systemic inflammation to cognitive function in midlife adults. Brain, Behavior, and Immunity, 48(April), 195-204. https://doi.org/10.1016/j.bbi.2015.03.015

Marteinsdottir, I., Ernerudh, J., Jonasson, L., Kristenson, M., \& Garvin, P. (2016). Psychological Resources Are Independently Associated with Markers of Inflammation in a Middle-Aged Community Sample. International Journal of Behavioral Medicine, 23(5), 611-620. https://doi.org/10.1007/s12529-016-9553-z

McAfoose, J., \& Baune, B. T. (2009). Evidence for a cytokine model of cognitive function. Neuroscience and Biobehavioral Reviews, 33(3), 355-366. https://doi.org/10.1016/j.neubiorev.2008.10.005

McGrew, R. W., \& Woodcock, K. S. (2001). Woodcock-Johnson III: Technical Manual. Riverside Publishing Company, Itasca, IL.

Miller, AH., Haroon, E., Raison, C. L., \& Felger, J. C. (2013). CYTOKINE TARGETS IN THE BRAIN: IMPACT ON NEUROTRANSMITTERS AND NEUROCIRCUITS. Depression and Anxiety, 30(4), 297-306. https://doi.org/10.1002/da.22084

Miller, AL, Jong, H., \& Lumeng, J. (2015). Obesity-Associated Biomarkers and Executive Function in Children. Pediatric Research, 77(0), 143-147. https://doi.org/10.1038/pr.2014.158.Obesity-Associated

Moore, S. A., Mckay, H. A., Macdonald, H., Nettlefold, L., Baxter-Jones, A. D. G., Cameron, N., \& Brasher, P. M. A. (2015). Enhancing a Somatic Maturity Prediction Model. Medicine \& Science in Sports \& Exercise, 47(8), 1755-1764. https://doi.org/10.1249/MSS.0000000000000588 
Moreno-López, L., Soriano-Mas, C., Delgado-Rico, E., Rio-Valle, J. S., \& VerdejoGarcía, A. (2012). Brain Structural Correlates of Reward Sensitivity and Impulsivity in Adolescents with Normal and Excess Weight. PLoS ONE, 7(11), e49185. https://doi.org/10.1371/journal.pone.0049185

Ng, M., Fleming, T., Robinson, M., Thomson, B., Graetz, N., Margono, C., ... Gakidou, E. (2014). Global, regional, and national prevalence of overweight and obesity in children and adults during 1980-2013: a systematic analysis for the Global Burden of Disease Study 2013. Lancet (London, England), 384(9945), 766-781. https://doi.org/10.1016/S0140-6736(14)60460-8

O’Shea, T. M., Shah, B., Allred, E. N., Fichorova, R. N., Kuban, K. C. K., Dammann, O., ... ELGAN Study Investigators. (2013). Inflammation-initiating illnesses, inflammation-related proteins, and cognitive impairment in extremely preterm infants. Brain, Behavior, and Immunity, 29, 104-112. https://doi.org/10.1016/j.bbi.2012.12.012

Reynolds, C. R., \& Kamphaus, R. W. (2004). Behavior assessment system for children (2nd. ed.). Circle Pines, MN: American Guidance Service, Inc. https://doi.org/10.1111/jsr.12055

Robinson, J. L., Bearden, C. E., Monkul, E. S., Tordesillas-Gutiérrez, D., Velligan, D. I., Frangou, S., \& Glahn, D. C. (2009). Fronto-temporal dysregulation in remitted bipolar patients: an fMRI delayed-non-match-to-sample (DNMS) study. Bipolar Disorders, 11(4), 351-360. https://doi.org/10.1111/j.1399-5618.2009.00703.x

Rose, J., Vassar, R., Cahill-Rowley, K., Hintz, S. R., \& Stevenson, D. K. (2015). Neonatal Biomarkers of Inflammation: Correlates of Early Neurodevelopment and 
Gait in Very-Low-Birth-Weight Preterm Children. American Journal of Perinatology, 33(1), 71-78. https://doi.org/10.1055/s-0035-1557106

Rosenblat, J. D., Cha, D. S., Mansur, R. B., \& McIntyre, R. S. (2014). Inflamed moods: A review of the interactions between inflammation and mood disorders. Progress in Neuro-Psychopharmacology and Biological Psychiatry, 53, 23-34. https://doi.org/10.1016/j.pnpbp.2014.01.013

Sanders, R. H., Han, A., Baker, J. S., \& Cobley, S. (2015). Childhood obesity and its physical and psychological co-morbidities: a systematic review of Australian children and adolescents. European Journal of Pediatrics, 174(6), 715-746. https://doi.org/10.1007/s00431-015-2551-3

Sartori, A. C., Vance, D. E., Slater, L. Z., \& Crowe, M. (2012). The impact of inflammation on cognitive function in older adults: implications for healthcare practice and research. The Journal of Neuroscience Nursing: Journal of the American Association of Neuroscience Nurses, 44(4), 206-217. https://doi.org/10.1097/JNN.0b013e3182527690

Satizabal, C. L., Zhu, Y. C., Mazoyer, B., Dufouil, C., \& Tzourio, C. (2012). Circulating IL-6 and CRP are associated with MRI findings in the elderly: The 3C$\begin{array}{llll}\text { Dijon } & \text { Study. } & \text { Neurology, } & \text { 78(10), }\end{array}$ https://doi.org/10.1212/WNL.0b013e318248e50f

Slopen, N., Kubzansky, L. D., \& Koenen, K. C. (2013). Internalizing and externalizing behaviors predict elevated inflammatory markers in childhood. Psychoneuroendocrinology, $38(12)$ $2854-2862$. https://doi.org/10.1016/j.psyneuen.2013.07.012 
Song, X.-W., Dong, Z.-Y., Long, X.-Y., Li, S.-F., Zuo, X.-N., Zhu, C.-Z., ... Zang, Y.F. (2011). REST: A Toolkit for Resting-State Functional Magnetic Resonance Imaging Data Processing. PLoS ONE, 6(9), e25031. https://doi.org/10.1371/journal.pone.0025031

Stroop, J. R. (1935). Studies of interference in serial verbal reactions. Journal of Experimental Psychology, 18(6), 643-662. https://doi.org/10.1037/h0054651

Taki, Y., Thyreau, B., Kinomura, S., Sato, K., Goto, R., Wu, K., ... Fukuda, H. (2013). Correlation between high-sensitivity C-reactive protein and brain gray matter volume in healthy elderly subjects. Human Brain Mapping, 34(10), 2418-2424. https://doi.org/10.1002/hbm.22073

Voltas, N., Arija, V., Hernández-Martínez, C., Jiménez-Feijoo, R., Ferré, N., \& Canals, J. (2017). Are there early inflammatory biomarkers that affect neurodevelopment in infancy? Journal of Neuroimmunology, 305, 42-50. https://doi.org/10.1016/j.jneuroim.2017.01.017

Windham, B. G., Simpson, B. N., Lirette, S., Bridges, J., Bielak, L., Peyser, P. A., ... Mosley, T. H. (2014). Associations between inflammation and cognitive function in African Americans and European Americans. Journal of the American Geriatrics Society, 62(12), 2303-2310. https://doi.org/10.1111/jgs.13165

Yirmiya, R., \& Goshen, I. (2011). Immune modulation of learning, memory, neural plasticity and neurogenesis. Brain, Behavior, and Immunity, 25(2), 181-213. https://doi.org/10.1016/j.bbi.2010.10.015

Zhang, J., Terreni, L., De Simoni MG, \& Dunn, A. J. (2001). Peripheral interleukin-6 administration increases extracellular concentrations of serotonin and the evoked 

308. Retrieved from http://www.ncbi.nlm.nih.gov/pubmed/11137624 
Table 1. Descriptive characteristics of the study sample.

\begin{tabular}{|c|c|c|c|}
\hline & All & Boys & Girls \\
\hline $\mathrm{N}$ & 107 & 63 & 44 \\
\hline \multicolumn{4}{|l|}{ Physical characteristics } \\
\hline Age (years) & $10.0 \pm 1.1$ & $10.2 \pm 1.2$ & $9.9 \pm 1.1$ \\
\hline Peak height velocity (years) & $-2.3 \pm 1.0$ & $-2.7 \pm 0.8$ & $-1.7 \pm 1.0$ \\
\hline Weight $(\mathrm{kg})$ & $55.9 \pm 11.0$ & $56.8 \pm 11.0$ & $54.5 \pm 11.1$ \\
\hline Height $(\mathrm{cm})$ & $144.1 \pm 8.4$ & $144.8 \pm 7.9$ & $143.0 \pm 9.1$ \\
\hline Body mass index $\left(\mathrm{kg} / \mathrm{m}^{2}\right)$ & $26.7 \pm 3.6$ & $26.9 \pm 3.7$ & $26.4 \pm 3.4$ \\
\hline Overweight/Obesity grade I/II/III (\%) & $26 / 44 / 20 / 10$ & $25 / 48 / 16 / 11$ & $27 / 39 / 25 / 9$ \\
\hline $\begin{array}{l}\text { Parental education university level: } \\
\text { Neither/One/Both parents }(\%)\end{array}$ & $64 / 19 / 17$ & $70 / 16 / 14$ & $57 / 23 / 20$ \\
\hline \multicolumn{4}{|l|}{ Inflammatory biomarkers } \\
\hline White blood cell $\left(10^{3} / \mu \mathrm{L}\right)(\mathrm{n}=101)$ & $7.3 \pm 1.7$ & $7.2 \pm 1.7$ & $7.6 \pm 1.6$ \\
\hline Interleukin-6 $(\mathrm{pg} / \mathrm{mL})^{\mathrm{a}}(\mathrm{n}=96)$ & $1.7 \pm 1.2$ & $1.7 \pm 1.3$ & $1.8 \pm 1.2$ \\
\hline Interleukin-1 $\beta(\mathrm{pg} / \mathrm{mL})^{\mathrm{a}}(\mathrm{n}=101)$ & $1.6 \pm 0.9$ & $1.5 \pm 0.9$ & $1.7 \pm 1.0$ \\
\hline $\begin{array}{l}\text { Tumor necrosis factor- } \alpha(\mathrm{pg} / \mathrm{mL})^{\mathrm{a}}(\mathrm{n}= \\
102)\end{array}$ & $4.1 \pm 1.5$ & $3.9 \pm 1.6$ & $4.3 \pm 1.4$ \\
\hline C-reactive protein $(\mathrm{mg} / \mathrm{L})^{\mathrm{a}}(\mathrm{n}=77)$ & $3.2 \pm 3.1$ & $3.4 \pm 3.4$ & $2.9 \pm 2.5$ \\
\hline \multicolumn{4}{|l|}{ Academic performance ${ }^{b}(n=106)$} \\
\hline Mathematics & $102.1 \pm 11.0$ & $102.8 \pm 11.8$ & $101.0 \pm 9.7$ \\
\hline Reading & $108.6 \pm 12.7$ & $109.0 \pm 11.0$ & $108.0 \pm 15.1$ \\
\hline Writing & $114.2 \pm 12.9$ & $113.2 \pm 12.1$ & $115.7 \pm 14.0$ \\
\hline Total achievement & $109.7 \pm 11.9$ & $109.7 \pm 11.0$ & $109.6 \pm 13.2$ \\
\hline \multicolumn{4}{|l|}{ Executive function } \\
\hline Cognitive flexibility ${ }^{c}$ & $20.0 \pm 6.5$ & $20.7 \pm 6.9$ & $19.1 \pm 6.0$ \\
\hline Cognitive inhibition ${ }^{\mathrm{a}, \mathrm{d}}$ & $-40.6 \pm 17.2$ & $-38.8 \pm 15.0$ & $-43.2 \pm 19.8$ \\
\hline Working memory ${ }^{\mathrm{a}, \mathrm{e}}$ & $15.2 \pm 6.7$ & $15.0 \pm 6.8$ & $15.4 \pm 6.8$ \\
\hline \multicolumn{4}{|c|}{ Behavioural and emotional functioning ${ }^{f}(n=106)$} \\
\hline Externalizing problems & $49.0 \pm 8.8$ & $49.1 \pm 9.1$ & $48.7 \pm 8.3$ \\
\hline Internalizing problems & $54.4 \pm 14.1$ & $52.9 \pm 14.0$ & $56.5 \pm 14.1$ \\
\hline Adaptive skills & $49.5 \pm 11.1$ & $48.8 \pm 10.7$ & $50.6 \pm 11.7$ \\
\hline Behavioural symptoms index & $50.8 \pm 11.1$ & $50.8 \pm 11.7$ & $50.9 \pm 10.2$ \\
\hline \multicolumn{4}{|l|}{ Brain volumes $\left(\mathrm{cm}^{3}\right)^{\mathrm{g}}(\mathrm{n}=99)$} \\
\hline Total gray matter & $793.0 \pm 66.4$ & $819.1 \pm 56.5$ & $754.5 \pm 61.4$ \\
\hline Total white matter & $406.0 \pm 47.5$ & $425.8 \pm 42.4$ & $376.7 \pm 38.9$ \\
\hline Total brain volume & $1199.0 \pm 106.4$ & $1244.9 \pm 88.9$ & $1131.2 \pm 93.7$ \\
\hline
\end{tabular}

Values are mean \pm standard deviation or percentages.

${ }^{a}$ Values were normalized using the Blom's formula before analysis, but non-transformed values are 
presented in the table.

${ }^{\mathrm{b}}$ Measured by the Bateria III Woodcock-Muñoz Tests of Achievement. Values based on standard Tscores with an average of 100 and standard deviations of 15 points.

${ }^{c}$ Measured by the Design fluency test as the total number of correct drawn designs.

${ }^{\mathrm{d}}$ Measured by Stroop test as the subtraction of the completion times of two tasks.

${ }^{\mathrm{e}}$ Measured by the Delayed nonmatch-to-sample task. Calculated as the quotient between reaction time and response accuracy.

${ }^{\mathrm{f}}$ Measured by the Behavior Assessment System for Children (BASC). Values based on standard Tscores with an average of 50 and standard deviations of 10 points.

${ }^{\mathrm{g}}$ Measured by Magnetic Resonance Imaging. 
[able 2. Associations between inflammatory biomarkers and brain health indicators in children with overweight/obesity.

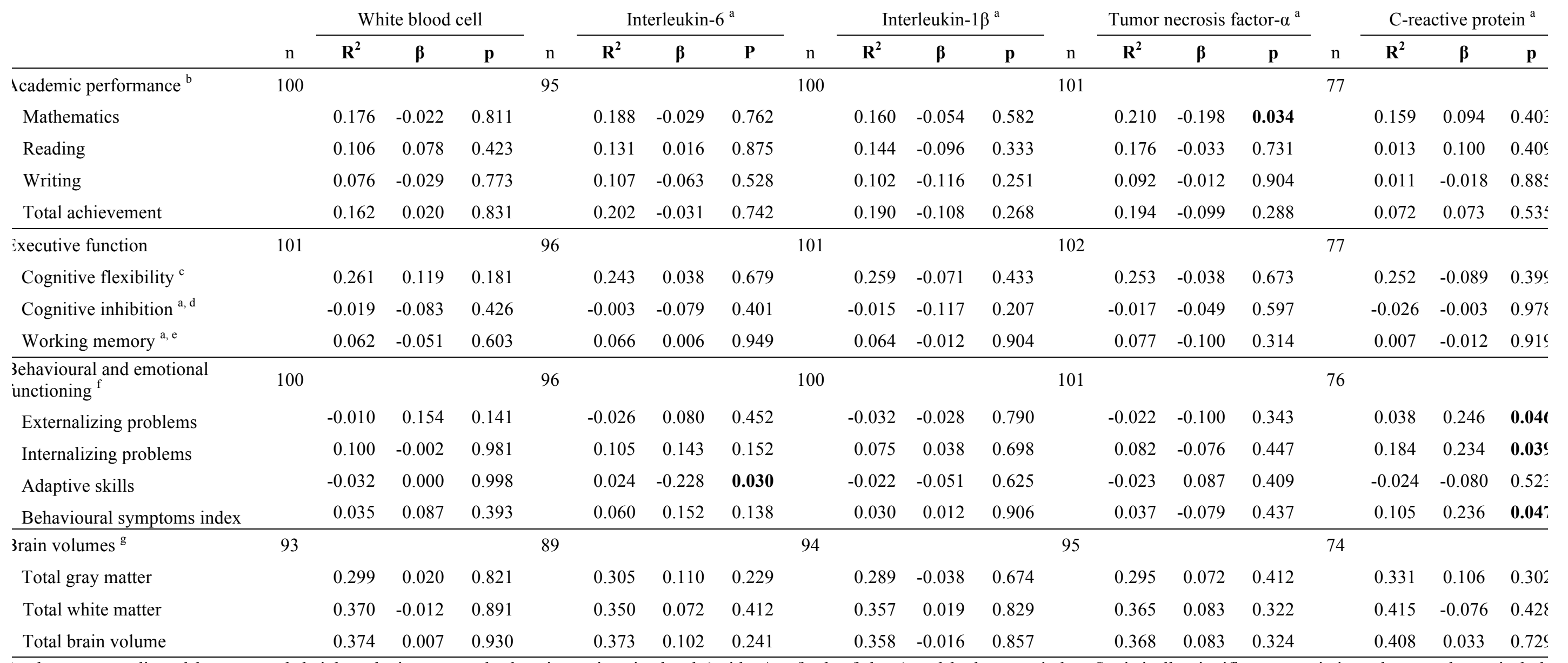

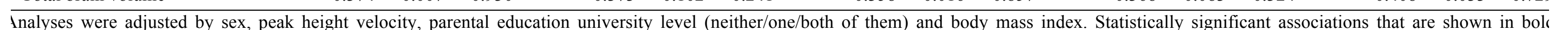

lisappeared when $\mathrm{p}$ values were adjusted for multiple comparisons using the Benjamini-Hochberg method. 
Blom's normalized values were used in the analysis.

Measured by the Bateria III Woodcock-Muñoz Tests of Achievement. Values based on standard T-scores with an average of 100 and standard deviations of 15 points.

Measured by the Design fluency test as the total number of correct drawn designs.

Measured by Stroop test as the subtraction of the completion times of two tasks. The original score was multiplied by -1 to invert the variable, so that a higher score indicates higher cognitive inhibition.

Measured by the Delayed nonmatch-to-sample task. Calculated as the quotient between reaction time and response accuracy. The original score was multiplied by -1 to invert the variable, so that a highe core indicates higher working memory.

Measured by the Behavior Assessment System for Children (BASC). Values based on standard T-scores with an average of 50 and standard deviations of 10 points.

Measured by Magnetic Resonance Imaging. 
Fig. 1. Brain regions showing positive associations of (A) white blood cell and (B) C-reactive protein with gray matter volume in children with overweight/obesity. Analyses were adjusted by sex, peak height velocity (years), parental education university level (neither/one/both) and body mass index $\left(\mathrm{kg} / \mathrm{m}^{2}\right)$. Each inflammatory biomarker was introduced in a separate model. Maps were thresholded using AlphaSim at $\mathrm{p}<0.001$ with $\mathrm{k}=44$ voxels for $\mathrm{WBC}$ and $\mathrm{k}=29$ for CRP, and surpassed Hayasaka correction. Anatomical coordinates (X, Y, Z) are given in Montreal Neurological Institute (MNI) Atlas space. The colour bar represents t-values, with lighter pink colour indicating higher significant association. Images are displayed in neurological convention; therefore, the right hemisphere corresponds to the right side in coronal displays. $\beta=$ standardized regression coefficient. ${ }^{\text {a }}$ Blom's normalized values were used in the analysis. ${ }^{*} \mathrm{p}<0.001$

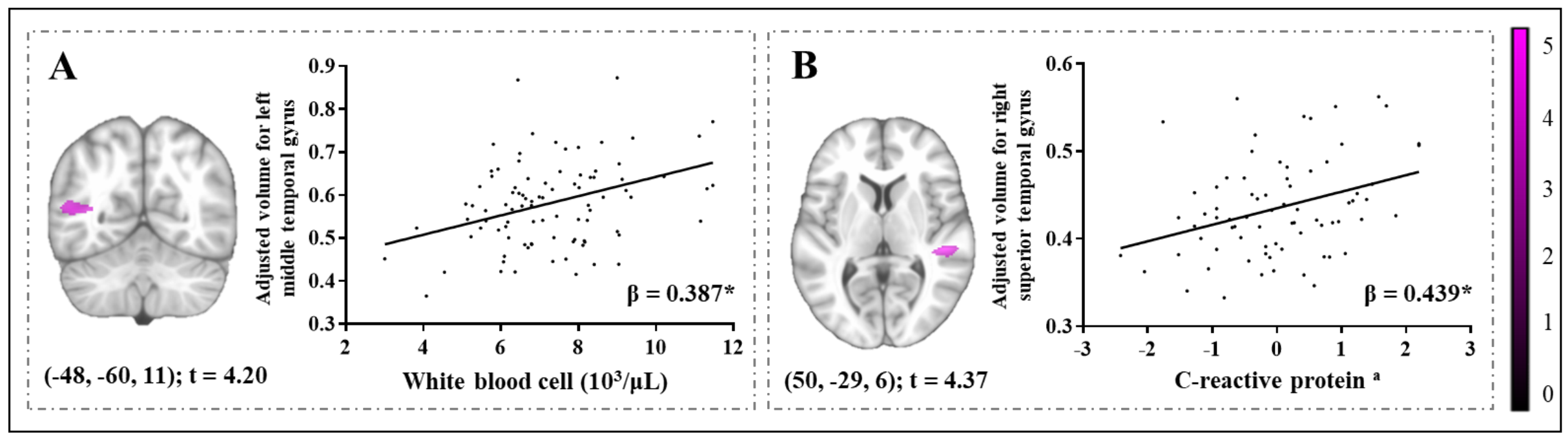


714 Fig. 2. Brain regions showing (A) positive associations of interleukin-6 and tumor necrosis factor- $\alpha$ with white matter volume and (B) negative association of C-reactive protein with white matter volume in children with overweight/obesity. Analyses were adjusted by sex, peak height velocity (years), parental education university level (neither/one/both) and body mass index $\left(\mathrm{kg} / \mathrm{m}^{2}\right)$. Each inflammatory biomarker was introduced in a separate model. Maps were thresholded using AlphaSim at $\mathrm{p}<0.001$ with $\mathrm{k}=81$ voxels for IL- 6 , $\mathrm{k}=62$ for TNF- $\alpha$, and $\mathrm{k}=82$ for CRP, and surpassed Hayasaka correction. Anatomical coordinates (X, Y, Z) are given in Montreal Neurological Institute (MNI) Atlas space. The colour bar represents t-values, with lighter green colour indicating higher significant association. Images are displayed in neurological convention; therefore, the right hemisphere corresponds to the right side in coronal displays. $\beta=$ standardized regression coefficient. ${ }^{a}$ Blom's normalized values were used in the analysis. ${ }^{*} \mathrm{p}<0.001$. 


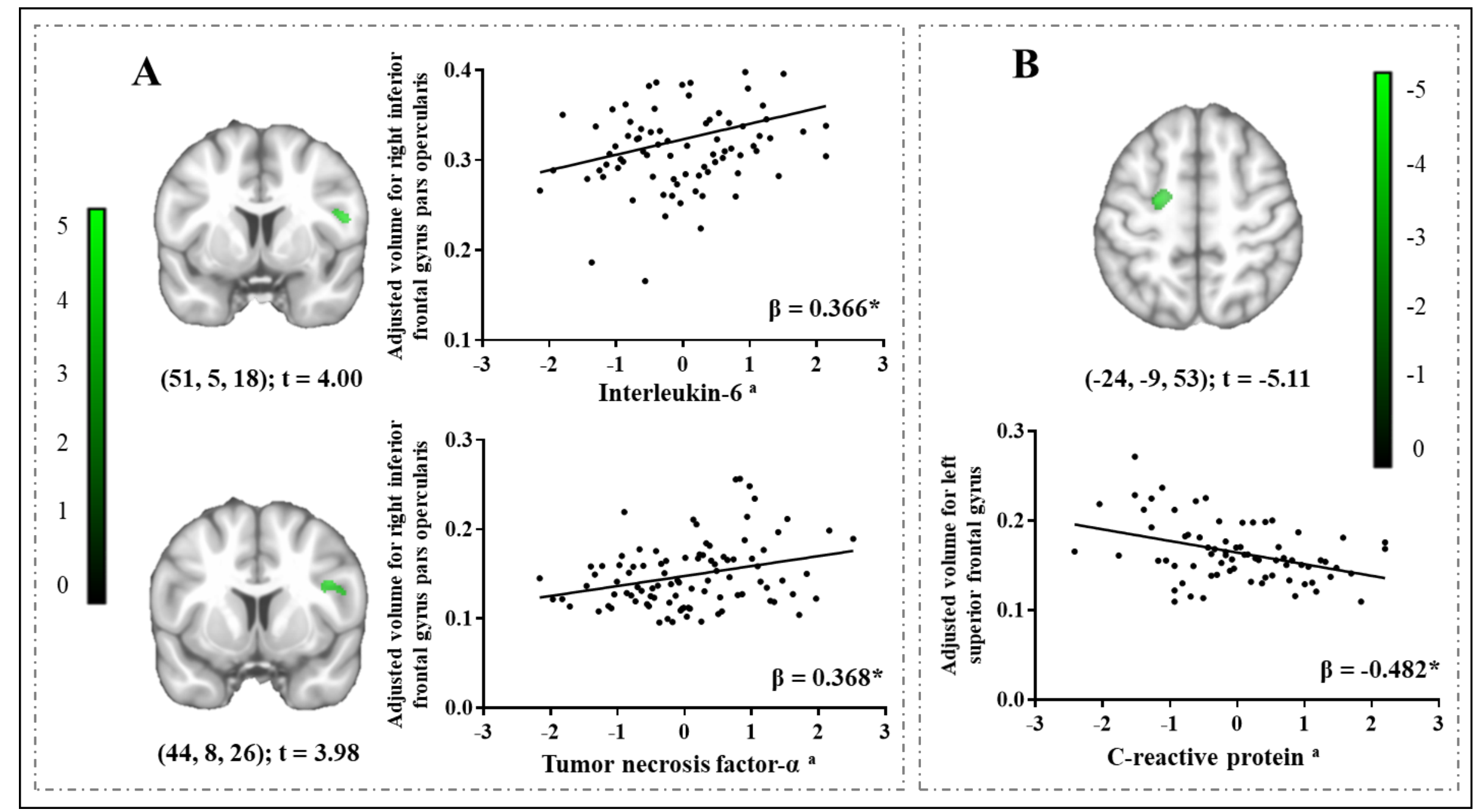


Table S1. Brain regions showing independent associations of inflammatory biomarkers with gray matter volume in children with overweight and obesity.

\section{Brain Regions $\left(\mathrm{mm}^{3}\right)$}

\section{Positive associations}

White blood cell

Middle temporal gyrus

C-reactive protein

Superior temporal gyrus

Supplementary motor cortex
Model 1

\begin{tabular}{ccccccc}
\multicolumn{10}{c}{ Model 1 } \\
\hline $\mathbf{x}$ & $\mathbf{y}$ & $\mathbf{z}$ & $\mathbf{t}$ & $\begin{array}{c}\text { Cluster } \\
\text { size }\end{array}$ & Hem & $\boldsymbol{\beta}$ \\
\hline
\end{tabular}

$\begin{array}{lllll}-48 & -60 & 11 & 4.20 & 24\end{array}$

$\begin{array}{ccc}50 & -29 & 6 \\ - & - & -\end{array}$

$\begin{array}{cc}6 & 4.37 \\ - & -\end{array}$

Model 1: Analyses were adjuste

sex, peak

\begin{tabular}{lllllll}
\multicolumn{10}{c}{ Model 2 } \\
\hline $\mathbf{x}$ & $\mathbf{y}$ & $\mathbf{z}$ & $\mathbf{t}$ & $\begin{array}{c}\text { Cluster } \\
\text { size }\end{array}$ & Hem & $\boldsymbol{\beta}$ \\
\hline
\end{tabular}

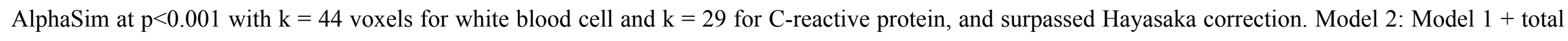

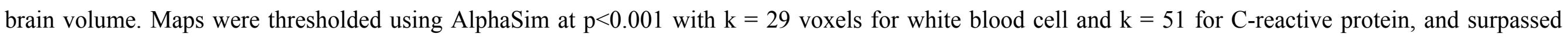

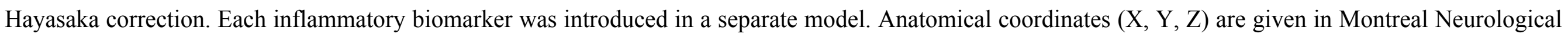
Institute (MNI) Atlas space. Hem, hemisphere; R, right; L, left. $\beta$ : standardized regression coefficient. 
Table S2. Brain regions showing independent associations of inflammatory biomarkers with white matter volume in children with overweight and obesity.

Model 1

\section{Brain Regions $\left(\mathrm{mm}^{3}\right)$}

Positive associations

Interleukin-6

Inferior frontal gyrus pars opercularis

Tumor necrosis factor- $\alpha$

Inferior frontal gyrus pars opercularis

\section{Negative associations}

C-reactive protein

Superior frontal gyrus

Superior frontal gyrus pars orbital

Superior frontal gyrus pars orbital

Middle cingulum

\begin{tabular}{lll}
\hline $\mathbf{y}$ & $\mathbf{z}$
\end{tabular}

\section{5}

44

1

\section{Cluster}

$\begin{array}{ccccc}-24 & -9 & 53 & -5.11 & 302 \\ - & - & - & - & - \\ - & - & - & - & - \\ - & - & - & - & -\end{array}$

Model 2

\begin{tabular}{lllllll}
\hline $\mathbf{x}$ & $\mathbf{y}$ & $\mathbf{z}$ & $\mathbf{t}$ & $\begin{array}{c}\text { Cluster } \\
\text { size }\end{array}$ & Hem & $\boldsymbol{\beta}$ \\
\hline
\end{tabular}

Model 1: Analyses were adjusted by sex, peak height velocity, parental education university level and body mass index. Maps were thresholded using AlphaSim at $\mathrm{p}<0.001$ with $\mathrm{k}=81$ voxels for interleukin- $6, \mathrm{k}=62$ for tumor necrosis factor- $\alpha$, and $\mathrm{k}=82$ for C-reactive protein, and surpassed Hayasaka correction. Model 2: Model $1+$ total brain volume. Maps were thresholded using AlphaSim at $\mathrm{p}<0.001$ with $\mathrm{k}=72$ voxels for interleukin- $6, \mathrm{k}=53$ for tumor necrosis factor- $\alpha$, and $\mathrm{k}=$ 87 for C-reactive protein, and surpassed Hayasaka correction. Each inflammatory biomarker was introduced in a separate model. Anatomical coordinates (X, Y, Z) are given in Montreal Neurological Institute (MNI) Atlas space. Hem, hemisphere; R, right; L, left. $\beta$ : standardized regression coefficient. 